\title{
A discussion on "On the solution of a class of fuzzy system of linear equations"
}

\author{
MAREK LANDOWSKI \\ Department of Mathematical Methods, Maritime University of Szczecin, Waly Chrobrego 1-2, 70-500 Szczecin, \\ Poland \\ e-mail: m.landowski@am.szczecin.pl
}

MS received 15 January 2018; revised 30 May 2018; accepted 18 June 2018; published online 17 November 2018

\begin{abstract}
The paper is a discussion on the results obtained by Salkuyeh in the article (Sādhānā 40(2):369-377, 2015). Salkuyeh considers the solution of the fuzzy system of linear equations (FSLE) and gives numerical examples where the results of FSLE are calculated. It was shown that the results by Salkuyeh are not full solutions and do not satisfy equivalent forms of the analysed FSLE. The paper presents a multidimensional approach to finding the solution of the FSLE. On the basis of the notation of the horizontal fuzzy number, a full solution that satisfies the FSLE and its equivalent forms is obtained.
\end{abstract}

Keywords. Fuzzy system of linear equations; fuzzy arithmetic; horizontal fuzzy number; RDM arithmetic.

\section{Introduction}

The paper is a discussion on the article by Salkuyeh [1] published in Sādhanā 2015 40(2): 369-377. In the paper [1], the author considers the solution of the fuzzy system of linear equations (FSLE) and presents examples with the results of the FSLE.

The FSLE defined in [1,2] as an $n \times n$ linear system (1) is considered:

$$
A \tilde{X}=\tilde{B}
$$

where $A=\left(a_{i j}\right)$ is an $n \times n$ real number coefficient matrix, $\tilde{B}=\left(\tilde{b}_{i}\right)$ is a column vector of fuzzy numbers, $\tilde{X}=\left(\tilde{x}_{j}\right)$ is an unknown vector of fuzzy numbers and $1 \leq i, j \leq n$.

In the paper it was shown that the results obtained in [1] are not full solutions. Moreover, the results in [1] do not satisfy equivalent forms of the FSLEs. The paper indicates examples with the crisp solutions of the FSLE that do not belong to the results generated by Salkuyeh. In paper [1] the author uses the standard fuzzy arithmetic (SFA) for the calculation (see [1] page 372), which is based on the standard interval arithmetic (SIA) [3]. In the SIA (or in the SFA), the operations are made only on the borders of intervals (or of fuzzy numbers). In more complicated problems (e.g., solution of the FSLE), this approach does not generate full solutions or leads to incorrect results $[4,5]$.

The approach using the horizontal fuzzy number (HFN) considered in paper [6] to find the solution of the FSLE leads to the results in a multidimensional space that have a form of the formula with HFN variables, see [6-9]. The obtained solution with HFN was called a direct solution. It was shown that the obtained direct solutions satisfy the FSLE and its equivalent forms. A span of the direct solution gives a secondary solution in the form of a fuzzy number. The direct solution is a full solution of the FSLE. Any crisp solution of the FSLE can be calculated from the direct solution with HFN variables. Also, using this method, a crisp system of linear equations (CSLE) that corresponds to the obtained crisp solution can be found. A method presented by Salkuyeh [1] does not offer a possibility to find the crisp solution and its CSLE on the basis of the obtained results.

\section{A discussion on the results of the FSLE by Salkuyeh [1]}

In this section it is shown that the results obtained by Salkuyeh in examples 1-3 in paper [1] are not full solutions.

In example 1, the CSLE can be obtained from the FSLE. For example for $r=0$, there exists the following CSLE (2):

$$
\begin{gathered}
2 x_{1}-3 x_{2}+x_{3}=7 \\
-x_{1}-3 x_{2}-x_{3}=-6 \\
-x_{1}+2 x_{2}+5 x_{3}=29
\end{gathered}
$$

where the crisp solution as shown in (3) is

$$
\begin{gathered}
x_{1}=19 / 49 \approx 0.39, \\
x_{2}=-5 / 49 \approx-0.1, \\
x_{3}=290 / 49 \approx 5.92 .
\end{gathered}
$$

However, the solution (3) does not belong to the solution presented in example 1 by Salkuyeh [1]. For $r=0$, the 
results of example 1 in [1] are intervals $\tilde{x}^{*}=([1,3] ;[1,5]$; $[2,4])$; thus $x_{1}=19 / 49 \approx 0.39 \notin[1,3], \quad x_{2}=-5 / 49 \approx$ $-0.1 \notin[1,5]$ and $x_{3}=290 / 49 \approx 5.92 \notin[2,4]$.

Considering example 2 from paper [1] for $r=0$, the CSLE (4) is obtained:

$$
\begin{gathered}
2 x_{1}-x_{2}+x_{3}=13 \\
-3 x_{1}+5 x_{2}+x_{3}=25 \\
-2 x_{1}-3 x_{2}+4 x_{3}=-23
\end{gathered}
$$

where the crisp solution is (5)

$$
x_{1}=42 / 5 \approx 8.4, x_{2}=9, x_{3}=26 / 5 \approx 5.2 .
$$

The crisp solution (5) is not present in the results calculated in example 2 in [1]; for $r=0, x_{1}=42 / 5 \approx 8.4 \notin[1,6]$, $x_{2}=9 \notin[2,5]$ and $x_{3}=26 / 5 \approx 5.2 \notin[1,3]$.

Similarly, from example 3 (paper [1]) the CSLE (6)

$$
\begin{gathered}
6 x_{1}-x_{2}-x_{3}=8 \\
-x_{1}+2 x_{2}-x_{3}=6 \\
-x_{1}-x_{2}+x_{3}=8
\end{gathered}
$$

with crisp solution (7)

$$
x_{1}=44, x_{2}=102, x_{3}=154
$$

are obtained. However, the crisp solution (7) does not belong to the result obtained in example 3 by Salkuyeh in paper [1]. For $r=0$ the result in example 3 in [1] is in the form of three intervals: $\tilde{x}^{*}=([-1,2] ;[1,4] ;[3,8])$; thus $\quad x_{1}=44 \notin[-1,2], \quad x_{2}=102 \notin[1,4], \quad x_{3}=154 \notin$ $[3,8]$.

Moreover, the obtained results in [1] do not satisfy equivalent forms of the considered FSLE.

The results presented by Salkuyeh (8) (example 1 from [1]) do not satisfy equivalent forms (9) and (10) of the considered FSLE.

$$
\tilde{x}_{1}=(1+r, 3-r), \tilde{x}_{2}=(1+2 r, 5-2 r), \tilde{x}_{3}=(2+r, 4-r) .
$$

$$
\begin{gathered}
2 \tilde{x}_{1}=(-11+9 r, 7-9 r)+3 \tilde{x}_{2}-\tilde{x}_{3}, \\
-\tilde{x}_{1}=(-22+8 r,-6-8 r)+3 \tilde{x}_{2}+\tilde{x}_{3}, \\
-\tilde{x}_{1}=(9+10 r, 29-10 r)-2 \tilde{x}_{2}-5 \tilde{x}_{3} . \\
2 \tilde{x}_{1}-3 \tilde{x}_{2}=(-11+9 r, 7-9 r)-\tilde{x}_{3}, \\
-\tilde{x}_{1}-3 \tilde{x}_{2}=(-22+8 r,-6-8 r)+\tilde{x}_{3}, \\
-\tilde{x}_{1}+2 \tilde{x}_{2}=(9+10 r, 29-10 r)-5 \tilde{x}_{3} .
\end{gathered}
$$

Next, it will be shown that the results calculated by Salkuyeh (8) do not satisfy the FSLE in the equivalent form (10).

Substituting results (8) into the left and right sides of the FSLE (10), with standard fuzzy arithmetic, leads to the following:

$$
\begin{aligned}
L_{1} & =2 \tilde{x}_{1}-3 \tilde{x}_{2}=2(1+r, 3-r)-3(1+2 r, 5-2 r) \\
& =(-13+8 r, 3-8 r), \\
L_{2} & =-\tilde{x}_{1}-3 \tilde{x}_{2}=-(1+r, 3-r)-3(1+2 r, 5-2 r) \\
& =(-18+7 r,-4-7 r), \\
L_{3} & =-\tilde{x}_{1}+2 \tilde{x}_{2}=-(1+r, 3-r)+2(1+2 r, 5-2 r) \\
& =(-1+5 r, 9-5 r), \\
R_{1} & =(-11+9 r, 7-9 r)-\tilde{x}_{3} \\
& =(-11+9 r, 7-9 r)-(2+r, 4-r) \\
& =(-15+10 r, 5-10 r), \\
R_{2} & =(-22+8 r,-6-8 r)+\tilde{x}_{3} \\
& =(-22+8 r,-6-8 r)+(2+r, 4-r) \\
& =(-20+9 r,-2-9 r), \\
R_{3} & =(9+10 r, 29-10 r)-5 \tilde{x}_{3} \\
& =(9+10 r, 29-10 r)-5(2+r, 4-r) \\
& =(-11+15 r, 19-15 r),
\end{aligned}
$$

hence $L_{i} \neq R_{i}, i=\{1,2,3\}$, the results (8) do not satisfy the FSLE (10).

Similarly, it can be shown that the results obtained in [1] for the given three examples do not satisfy equivalent forms of considered FSLEs.

\section{Introduction to HFN}

In paper [1], definitions of the FSLE, fuzzy number and parametric form of fuzzy number are presented.

A new multidimensional horizontal approach to fuzzy sets was presented in [6-9]. The standard definition of the fuzzy set considers the membership vertically. The new multidimensional approach considers the fuzzy set horizontally. Using variable $\alpha$ from interval $[0,1]$, the membership function is described as a set of points from the given intervals for each $r$-cut. This approach to the fuzzy membership function is called a horizontal membership function (HMF), while this approach applied to the fuzzy number is called a HFN.

To introduce the horizontal concept of the fuzzy number, first an interval notation in Relative Distance Measure interval arithmetic (RDMIA) should be described. The detailed concept of RDMIA and differences between RDMIA and standard interval arithmetic (SIA) are presented in [5, 10-12]. In the RDMIA approach, an element $x$ of interval $X=[\underline{x}, \bar{x}]$ is described using RDM variable $\alpha_{x} \in[0,1]$ as $x=\underline{x}+\alpha_{x}$ $(\bar{x}-\underline{x})$. The interval $X=[\underline{x}, \bar{x}]$ has a form of set (11)

$$
X=\left\{x: x=\underline{x}+\alpha_{x}(\bar{x}-\underline{x}), \alpha_{x} \in[0,1]\right\} .
$$

Based on the concept of RDMIA, the fuzzy number called HFN is defined [6].

Definition 1 (HFN) A HFN $\tilde{U}$ of an arbitrary fuzzy number $(\underline{u}(r), \bar{u}(r))$ is a set of numbers $\tilde{u}$ for $\alpha_{\tilde{u}} \in[0,1]$ given by (12): 


$$
\tilde{U}=\left\{\tilde{u}: \tilde{u}=\underline{u}(r)+\alpha_{\tilde{u}}(\bar{u}(r)-\underline{u}(r)), r, \alpha_{\tilde{u}} \in[0,1]\right\} .
$$

For HFN (12), the arithmetic called horizontal fuzzy arithmetic (HFA) is defined [6].

Definition 2 (HFA) For any two fuzzy numbers $\tilde{U}_{1}=$ $\left(\underline{u}_{1}(r), \bar{u}_{1}(r)\right)$ and $\tilde{U}_{2}=\left(\underline{u}_{2}(r), \bar{u}_{2}(r)\right)$ in notation of the HFN, the results of the basic arithmetic operations $\odot \in\{+,-, \times, /\}$ are defined as a set (13), $0 \notin \tilde{U}_{2}$ for operation / :

$$
\begin{gathered}
\tilde{U}_{1} \odot \tilde{U}_{2} \\
=\left\{\tilde{u}_{1} \odot \tilde{u}_{2}: \tilde{u}_{1} \odot \tilde{u}_{2}=\left[\underline{u}_{1}(r)+\alpha_{\tilde{u}_{1}}\left(\bar{u}_{1}(r)-\underline{u}_{1}(r)\right)\right]\right. \\
\left.\odot\left[\underline{u}_{2}(r)+\alpha_{\tilde{u}_{2}}\left(\bar{u}_{2}(r)-\underline{u}_{2}(r)\right)\right], r, \alpha_{\tilde{u}_{1}}, \alpha_{\tilde{u}_{2}} \in[0,1]\right\} .
\end{gathered}
$$

The HFN (12) can also be defined as (14):

$$
\tilde{u}=\tilde{u}\left(r, \alpha_{\tilde{u}}\right)=\underline{u}(r)+\alpha_{\tilde{u}}(\bar{u}(r)-\underline{u}(r)),
$$

where $r, \alpha_{\tilde{u}} \in[0,1]$.

A trapezoidal fuzzy number $\tilde{X}=(a+(b-a) r$; $d+(c-d) r)$, where $r \in[0,1], a \leq b \leq c \leq d$, in HFN notation has the form of (15):

$$
\tilde{x}\left(r, \alpha_{\tilde{x}}\right)=a+(b-a) r+\alpha_{\tilde{x}}[d-a+r(c-d-b+a)],
$$

where $r, \alpha_{\tilde{x}} \in[0,1]$.

For $b=c$ in formula (15) a triangular fuzzy number is obtained.

The presented result in (13) is called a direct solution. Calculating the span of the direct solution, the secondary solution in the form of the fuzzy number is obtained.

Definition 3 (Span) The span of the direct solution of the operation $\odot \in\{+,-, \times . /\}$ on the HFN $\tilde{u}_{1}\left(r, \alpha_{\tilde{u}_{1}}\right)$ and $\tilde{u}_{2}\left(r, \alpha_{\tilde{u}_{2}}\right)$ is defined as in Eq. (16):

$$
\operatorname{span}\left(\tilde{u}_{1} \odot \tilde{u}_{2}\right)=\left(\min _{\alpha_{\tilde{u}_{1}}, \alpha_{\tilde{u}_{2}}}\left(\tilde{u}_{1} \odot \tilde{u}_{2}\right), \max _{\alpha_{\tilde{u}_{1}}, \alpha_{\tilde{u}_{2}}}\left(\tilde{u}_{1} \odot \tilde{u}_{2}\right)\right)
$$

where $r, \alpha_{\tilde{u}_{1}}, \alpha_{\tilde{u}_{2}} \in[0,1]$.

In the HFA, operations are made on each element in the considered fuzzy sets; hence the HFA satisfies the arithmetic properties as in the standard arithmetic on a real set of numbers.

\section{Numerical examples}

In the following section, three numerical examples from [1] with the use of the HFN are solved.

Example 1 Let us consider the FSLE (17), where $r \in[0,1]$ :

$$
\begin{gathered}
2 \tilde{x}_{1}-3 \tilde{x}_{2}+\tilde{x}_{3}=(-11+9 r, 7-9 r), \\
-\tilde{x}_{1}-3 \tilde{x}_{2}-\tilde{x}_{3}=(-22+8 r,-6-8 r), \\
-\tilde{x}_{1}+2 \tilde{x}_{2}+5 \tilde{x}_{3}=(9+10 r, 29-10 r) .
\end{gathered}
$$

The FSLE (17) in the HFN notation has the form of (18):

$$
\begin{aligned}
& 2 \tilde{x}_{1}-3 \tilde{x}_{2}+\tilde{x}_{3}=-11+9 r+18 \alpha_{1}(1-r) \\
& -\tilde{x}_{1}-3 \tilde{x}_{2}-\tilde{x}_{3}=-22+8 r+16 \alpha_{2}(1-r) \\
& -\tilde{x}_{1}+2 \tilde{x}_{2}+5 \tilde{x}_{3}=9+10 r+20 \alpha_{3}(1-r)
\end{aligned}
$$

where $r, \alpha_{1}, \alpha_{2}, \alpha_{3} \in[0,1]$.

The direct solution with HFN variable of the FSLE (18) is presented in (19), where $r, \alpha_{1}, \alpha_{2}, \alpha_{3} \in[0,1]$.

$$
\begin{aligned}
\tilde{x}_{1}\left(r, \alpha_{1}, \alpha_{2}, \alpha_{3}\right)= & \left(-234 \alpha_{1}(r-1)+272 \alpha_{2}(r-1)\right. \\
& \left.+120 \alpha_{3}(r-1)-79 r+177\right) / 49, \\
\tilde{x}_{2}\left(r, \alpha_{1}, \alpha_{2}, \alpha_{3}\right)= & \left(108 \alpha_{1}(r-1)+176 \alpha_{2}(r-1)\right. \\
& \left.+20 \alpha_{3}(r-1)-152 r+299\right) / 49, \\
\tilde{x}_{3}\left(r, \alpha_{1}, \alpha_{2}, \alpha_{3}\right)= & \left(-90 \alpha_{1}(r-1)-16 \alpha_{2}(r-1)\right. \\
& \left.-180 \alpha_{3}(r-1)+143 r+4\right) / 49,
\end{aligned}
$$

The obtained solution with the HFN satisfies the FSLE. Substituting the direct solution (19) to the left sides of equations of the FSLE (17), the fuzzy numbers on the right sides are obtained; therefore

$$
\begin{aligned}
& L_{1}= 2 \tilde{x}_{1}-3 \tilde{x}_{2}+\tilde{x}_{3} \\
&= 2\left[-234 \alpha_{1}(r-1)+272 \alpha_{2}(r-1)+120 \alpha_{3}(r-1)\right. \\
&-79 r+177] / 49-3\left[108 \alpha_{1}(r-1)+176 \alpha_{2}(r-1)\right. \\
&\left.+20 \alpha_{3}(r-1)-152 r+299\right] / 49+\left[-90 \alpha_{1}(r-1)\right. \\
&\left.-16 \alpha_{2}(r-1)-180 \alpha_{3}(r-1)+143 r+4\right] / 49 \\
&= {\left[-882 \alpha_{1}(r-1)+441 r-539\right] / 49 } \\
&=-11+9 r+18 \alpha_{1}(1-r) \\
&=(-11+9 r, 7-9 r) \\
&= R_{1}, \\
& L_{2}=-\tilde{x}_{1}-3 \tilde{x}_{2}-\tilde{x}_{3} \\
&=-\left[-234 \alpha_{1}(r-1)+272 \alpha_{2}(r-1)+120 \alpha_{3}(r-1)\right. \\
&-79 r+177] / 49-3\left[108 \alpha_{1}(r-1)+176 \alpha_{2}(r-1)\right. \\
&\left.+20 \alpha_{3}(r-1)-152 r+299\right] / 49-\left[-90 \alpha_{1}(r-1)\right. \\
&\left.-16 \alpha_{2}(r-1)-180 \alpha_{3}(r-1)+143 r+4\right] / 49 \\
&= {\left[-784 \alpha_{2}(r-1)+392 r-1078\right] / 49 } \\
&=-22+8 r+16 \alpha_{2}(1-r) \\
&=(-22+8 r,-6-8 r) \\
&= R 2 \\
& L_{3}=-\tilde{x}_{1}+2 \tilde{x}_{2}+5 \tilde{x}_{3} \\
&=-\left[-234 \alpha_{1}(r-1)+272 \alpha_{2}(r-1)+120 \alpha_{3}(r-1)\right. \\
&-79 r+177] / 49+2\left[108 \alpha_{1}(r-1)+176 \alpha_{2}(r-1)\right. \\
&\left.+20 \alpha_{3}(r-1)-152 r+299\right] / 49+5\left[-90 \alpha_{1}(r-1)\right. \\
&\left.-16 \alpha_{2}(r-1)-180 \alpha_{3}(r-1)+143 r+4\right] / 49 \\
&= {\left[-980 \alpha_{3}(r-1)+490 r+441\right] / 49 } \\
&= 9+10 r+20 \alpha_{3}(1-r) \\
&=(9+10 r, 29-10 r) \\
&= R 3 . \\
& \hline
\end{aligned}
$$


It is easy to show that the direct solution (19) also satisfies the equivalent forms of the FSLE (17).

The secondary solution (span) of the direct solution (19) in the form of fuzzy numbers is presented as (20), $r \in[0,1]:$

$$
\begin{aligned}
& \operatorname{span}\left(\tilde{x}_{1}\right)=(-4.39+6.39 r, 8.39-6.39 r), \\
& \operatorname{span}\left(\tilde{x}_{2}\right)=(-0.1+3.1 r, 6.1-3.1 r), \\
& \operatorname{span}\left(\tilde{x}_{3}\right)=(0.08+2.92 r, 5.92-2.92 r) .
\end{aligned}
$$

The obtained fuzzy numbers (20) are different from the results calculated by Salkuyeh [1] in example 1 .

The crisp solution (3) and the CSLE (2) can be calculated from the direct solution (19) and the FSLE (18) for $r=0$ and $\alpha_{1}=\alpha_{2}=\alpha_{3}=1$.

Example 2 Let us consider the FSLE (21), where $r \in[0,1]$ :

$$
\begin{gathered}
2 \tilde{x}_{1}-\tilde{x}_{2}+\tilde{x}_{3}=(-2+9 r, 13-6 r), \\
-3 \tilde{x}_{1}+5 \tilde{x}_{2}+\tilde{x}_{3}=(-7+12 r, 25-20 r), \\
-2 \tilde{x}_{1}-3 \tilde{x}_{2}+4 \tilde{x}_{3}=(-23+14 r, 4-13 r) .
\end{gathered}
$$

The FSLE (21) where the fuzzy numbers have a form of the HFN is given by the FSLE (22):

$$
\begin{gathered}
2 \tilde{x}_{1}-\tilde{x}_{2}+\tilde{x}_{3}=-2+9 r+15 \alpha_{1}(1-r) \\
-3 \tilde{x}_{1}+5 \tilde{x}_{2}+\tilde{x}_{3}=-7+12 r+32 \alpha_{2}(1-r) \\
-2 \tilde{x}_{1}-3 \tilde{x}_{2}+4 \tilde{x}_{3}=-23+14 r+27 \alpha_{3}(1-r)
\end{gathered}
$$

where $r, \alpha_{1}, \alpha_{2}, \alpha_{3} \in[0,1]$.

The direct solution with the HFN has the form of (23):

$$
\begin{aligned}
\tilde{x}_{1}\left(r, \alpha_{1}, \alpha_{2}, \alpha_{3}\right)= & \left(-69 \alpha_{1}(r-1)-6.4 \alpha_{2}(r-1)\right. \\
& \left.+32.4 \alpha_{3}(r-1)+27 r+17\right) / 11 \\
\tilde{x}_{2}\left(r, \alpha_{1}, \alpha_{2}, \alpha_{3}\right)= & \left(-30 \alpha_{1}(r-1)-64 \alpha_{2}(r-1)\right. \\
& \left.+27 \alpha_{3}(r-1)+28 r+5\right) / 11, \\
\tilde{x}_{3}\left(r, \alpha_{1}, \alpha_{2}, \alpha_{3}\right)= & \left(-57 \alpha_{1}(r-1)-51.2 \alpha_{2}(r-1)\right. \\
& \left.-37.8 \alpha_{3}(r-1)+73 r-51\right) / 11,
\end{aligned}
$$

where $r, \alpha_{1}, \alpha_{2}, \alpha_{3} \in[0,1]$

Substituting the direct solution (23) for the FSLE (21), it will be shown that the solution (23) satisfies the considered FSLE. Therefore

$$
\begin{aligned}
L_{1}= & 2 \tilde{x}_{1}-\tilde{x}_{2}+\tilde{x}_{3} \\
= & 2\left(-69 \alpha_{1}(r-1)-6.4 \alpha_{2}(r-1)+32.4 \alpha_{3}(r-1)\right. \\
& +27 r+17) / 11-\left(-30 \alpha_{1}(r-1)-64 \alpha_{2}(r-1)\right. \\
& \left.+27 \alpha_{3}(r-1)+28 r+5\right) / 11+\left(-57 \alpha_{1}(r-1)\right. \\
& \left.-51.2 \alpha_{2}(r-1)-37.8 \alpha_{3}(r-1)+73 r-51\right) / 11 \\
= & \left(-165 \alpha_{1}(r-1)+99 r-22\right) / 11 \\
= & -2+9 r+15 \alpha_{1}(1-r) \\
= & (-2+9 r, 13-6 r) \\
= & R_{1},
\end{aligned}
$$

$$
\begin{aligned}
L_{2}= & -3 \tilde{x}_{1}+5 \tilde{x}_{2}+\tilde{x}_{3} \\
= & -3\left(-69 \alpha_{1}(r-1)-6.4 \alpha_{2}(r-1)+32.4 \alpha_{3}(r-1)\right. \\
& +27 r+17) / 11+5\left(-30 \alpha_{1}(r-1)-64 \alpha_{2}(r-1)\right. \\
& \left.+27 \alpha_{3}(r-1)+28 r+5\right) / 11+\left(-57 \alpha_{1}(r-1)\right. \\
& \left.-51.2 \alpha_{2}(r-1)-37.8 \alpha_{3}(r-1)+73 r-51\right) / 11 \\
= & \left(-352 \alpha_{2}(r-1)+132 r-77\right) / 11 \\
= & -7+12 r+32 \alpha_{2}(1-r) \\
= & (-7+12 r, 25-20 r) \\
= & R_{2}, \\
L_{3}= & -2 \tilde{x}_{1}-3 \tilde{x}_{2}+4 \tilde{x}_{3} \\
= & -2\left(-69 \alpha_{1}(r-1)-6.4 \alpha_{2}(r-1)+32.4 \alpha_{3}(r-1)\right. \\
& +27 r+17) / 11-3\left(-30 \alpha_{1}(r-1)-64 \alpha_{2}(r-1)\right. \\
& \left.+27 \alpha_{3}(r-1)+28 r+5\right) / 11+4\left(-57 \alpha_{1}(r-1)\right. \\
& \left.-51.2 \alpha_{2}(r-1)-37.8 \alpha_{3}(r-1)+73 r-51\right) / 11 \\
= & \left(-297 \alpha_{3}(r-1)+154 r-253\right) / 11 \\
= & -23+14 r+27 \alpha_{3}(1-r) \\
= & (-23+14 r, 4-13 r) \\
= & R 3 .
\end{aligned}
$$

Thus $L=R$. Also, similarly, it can be checked that the direct solution (23) satisfies equivalent forms of the FSLE (21).

The secondary solution in the form of fuzzy numbers obtained as a span of the direct solution (23) has the form of (24), where $r \in[0,1]$ :

$$
\begin{aligned}
& \operatorname{span}\left(\tilde{x}_{1}\right)=(-1.4+5.4 r, 8.4-4.4 r), \\
& \operatorname{span}\left(\tilde{x}_{2}\right)=(-2+5 r, 9-6 r), \\
& \operatorname{span}\left(\tilde{x}_{3}\right)=(-4.64+6.64 r, 8.64-6.64 r) .
\end{aligned}
$$

The obtained secondary solution in the form of fuzzy numbers is different from the fuzzy numbers calculated by Salkuyeh in example 2 in the article [1].

Substituting $r=0, \alpha_{1}=\alpha_{2}=1$ and $\alpha_{3}=0$ into the FSLE (22) and the direct solution (23), the CSLE (4) and its crisp solution (5) are obtained.

Example 3 Let us consider the FSLE (25), where $r \in[0,1]$ :

$$
\begin{gathered}
6 \tilde{x}_{1}-\tilde{x}_{2}-\tilde{x}_{3}=(-18+16 r, 8-10 r), \\
-\tilde{x}_{1}+2 \tilde{x}_{2}-\tilde{x}_{3}=(-8+8 r, 6-6 r), \\
-\tilde{x}_{1}-\tilde{x}_{2}+\tilde{x}_{3}=(-3+4 r, 8-7 r) .
\end{gathered}
$$

The FSLE (25) expressed in the notation with HFNs is presented as (26), where $r, \alpha_{1}, \alpha_{2}, \alpha_{3} \in[0,1]$ :

$$
\begin{aligned}
& 6 \tilde{x}_{1}-\tilde{x}_{2}-\tilde{x}_{3}=-18+16 r+26 \alpha_{1}(1-r), \\
& -\tilde{x}_{1}+2 \tilde{x}_{2}-\tilde{x}_{3}=-8+8 r+14 \alpha_{2}(1-r), \\
& -\tilde{x}_{1}-\tilde{x}_{2}+\tilde{x}_{3}=-3+4 r+11 \alpha_{3}(1-r) .
\end{aligned}
$$

The obtained direct solution of the FSLE (25) in HFN notation has the form of (27), where $r, \alpha_{1}, \alpha_{2}, \alpha_{3} \in[0,1]$ : 


$$
\begin{aligned}
\tilde{x}_{1}\left(r, \alpha_{1}, \alpha_{2}, \alpha_{3}\right)= & -26 \alpha_{1}(r-1)-28 \alpha_{2}(r-1) \\
& -33 \alpha_{3}(r-1)+44 r-43, \\
\tilde{x}_{2}\left(r, \alpha_{1}, \alpha_{2}, \alpha_{3}\right)= & -52 \alpha_{1}(r-1)-70 \alpha_{2}(r-1) \\
& -77 \alpha_{3}(r-1)+100 r-97, \\
\tilde{x}_{3}\left(r, \alpha_{1}, \alpha_{2}, \alpha_{3}\right)= & -78 \alpha_{1}(r-1)-98 \alpha_{2}(r-1) \\
& -121 \alpha_{3}(r-1)+148 r-143 .
\end{aligned}
$$

As in the previous examples, it is easy to show that the obtained direct solution (27) satisfies the FSLE (25) and its equivalent forms. Substituting the direct solution (27) to the left side of the FSLE (25), the right side of the FSLE will be obtained. A similar calculation can be shown with the equivalent forms of the FSLE (25). The proof is left to the reader.

The secondary solution in the form of fuzzy numbers of the direct solution (27) is presented by (28), $r \in[0,1]$.

$$
\begin{aligned}
& \operatorname{span}\left(\tilde{x}_{1}\right)=(-43+44 r, 44-43 r) \\
& \operatorname{span}\left(\tilde{x}_{2}\right)=(-97+100 r, 102-99 r) \\
& \operatorname{span}\left(\tilde{x}_{3}\right)=(-143+148 r, 154-149 r)
\end{aligned}
$$

The obtained fuzzy numbers (28) are different from the fuzzy numbers in example 3 presented by Salkuyeh in the paper [1].

The CSLE (6) and its crisp solution (7) can be calculated from the FSLE (26) and the direct solution (27) for $r=0$ and $\alpha_{1}=\alpha_{2}=\alpha_{3}=1$.

\section{Conclusion}

The paper shows that the results calculated by Salkuyeh in [1] are not full solutions and do not satisfy equivalent forms of the FSLE. The approach presented in the paper, using the HFN, provides a full solution of the FSLE. It is easy to show that the solutions obtained with the method that use the HFN satisfy the FSLE and its equivalent forms. Moreover, using the direct solution with HFNs, any crisp solution of FSLE - and CSLE corresponding to this crisp solution - can be calculated. The method presented by Salkuyeh [1] does not possess this property. Using the direct solution with HFN, the secondary solution in the form of the fuzzy number can be calculated.

\section{References}

[1] Salkuyeh D K 2015 On the solution of a class of fuzzy system of linear equations. Sadhana Acad. Proc. Eng. Sci. 40: $369-377$

[2] Friedman M, Ming M and Kandel A 1998 Fuzzy linear systems. Fuzzy Sets Syst. 96: 201-209

[3] Moore R E, Kearfott R B and Cloud J M 2009 Introduction to interval analysis. Philadelphia: SIAM

[4] Dymowa L 2011 Soft computing in economics and finance. Berlin-Heidelberg: Springer

[5] Landowski M 2015 Differences between Moore and RDM interval arithmetic. In: Proceedings of the 7th IEEE International Conference on Intelligent Systems, IEEE IS'2014. Cham: Springer, pp. 331-340

[6] Landowski M 2018 Method with horizontal fuzzy numbers for solving real fuzzy linear systems. Soft Comput. 1-13, https://doi.org/10.1007/s00500-018-3290-y

[7] Piegat A and Landowski M 2015 Horizontal membership function and examples of its applications. Int. J. Fuzzy Syst. 17: 22-30

[8] Piegat A and Landowski M 2017 Fuzzy arithmetic type 1 with horizontal membership functions. In: Kreinovich V (Ed) Uncertainty modeling. Studies in computational intelligence. Cham: Springer, pp. 233-250

[9] Piegat A and Landowski M 2018 Is fuzzy number the right result of arithmetic operations on fuzzy numbers? In: Proceedings of the 10th Conference of the European Society for Fuzzy Logic and Technology EUSFLAT 2017. Cham: Springer, pp. 181-194

[10] Piegat A and Landowski M 2012 Is the conventional interval arithmetic correct? J. Theor. Appl. Comput. Sci. 6: 27-44

[11] Piegat A and Landowski M 2013 Two interpretations of multidimensional RDM interval arithmetic-multiplication and division. Int. J. Fuzzy Syst. 15: 488-496

[12] Piegat A and Landowski M 2017 Is an interval the right result of arithmetic operations on intervals? Int. J. Appl. Math. Comput. Sci. 27: 575-590 